\section{NSCLC: EGFR-TKI gegen T790M-Mutation}

\section{Osimertinib inhibiert selektiv die EGFR-T790M-Tyrosinkinase. Nun wurde die Wirksamkeit der Substanz bei Patienten mit nichtkleinzelligem Lungen- karzinom (NSCLC) untersucht, deren Tumor die T790M-Mutation im EGFR(„epidermal growth factor receptor")-Gen aufwies. Im Vergleichsarm wurde eine platinbasierte Therapie plus Pemetrexed eingesetzt.}

Etwa wa $60 \%$ der Patienten mit NSCLC, bei denen es während der Therapie mit einem EGFR-Tyrosinkinaseinhibitor (TKI) zur Progression kommt, weisen eine T790M-Punktmutation im EGFRGen auf. Diese Mutation reduziert die Bindung von EGFR-TKI der ersten und zweiten Generation an EGFR.

An der Phase-III-Studie AURA3 nahmen 419 Patienten mit T790M-positivem fortgeschrittenem NSCLC teil, bei denen es nach der Erstlinientherapie mit einem EGFR-TKI zur Krankheitsprogression gekommen war. Sie erhielten im Verhältnis 2:1 entweder Osimertinib (oral $80 \mathrm{mg}$ 1-mal täglich) oder Pemetrexed (500 mg/m² intravenös) plus Carboplatin oder Cisplatin alle 3 Wochen über bis zu 6 Zyklen. Eine Erhaltungstherapie mit Pemetrexed war erlaubt. Der primäre Endpunkt war das progressionsfreie Überleben (PFS).

Die mediane Beobachtungsdauer betrug 8,3 Monate. Das PFS war unter Osimertinib signifikant länger als unter der platinbasierten Therapie plus Pemetrexed (median 10,1 vs. 4,4 Monate; Hazard Ratio [HR] 0,30, 95\%-Konfidenzintervall [95\%-KI] 0,23-0,41; p < 0,001). Nach 6 Monaten betrug die Rate für das PFS 69 versus $37 \%$ und nach 12 Monaten 44 versus $10 \%$. Hinsichtlich des PFS war der TKI in allen Subgruppen überlegen, auch bei Patienten mit ZNS-Metastasen ( $\mathrm{n}=144$; median 8,5 vs. 4,2 Monate; HR $0,32,95 \%$-KI $0,21-0,49)$.
Die Rate des objektiven Ansprechens war unter Osimertinib ebenfalls signifikant besser als unter der Platintherapie plus Pemetrexed (71 vs. $31 \%$; Odds Ratio 5,$39 ; \mathrm{p}<0,001$ ).

Unerwünschte Ereignisse vom Grad $\geq 3$ traten unter Osimertinib seltener auf als unter der Platintherapie plus Pemetrexed ( 23 vs. $47 \%$ ). Die häufigsten unerwünschten Ereignisse unter Osimertinib waren Diarrhö (41\%), Rash (34\%), trockene Haut (23\%) und Paronychie (22\%).

Fazit: Osimertinib hatte bei Patienten mit T790M-positivem fortgeschrittenem NSCLC, bei denen es unter der Erstlinientherapie mit einem EGFR-TKI zur Progression gekommen war, eine signifikant höhere Wirksamkeit als die Platintherapie in Kombination mit Pemetrexed. Die Überlegenheit des TKI war auch bei Patienten mit ZNS-Metastasen zu verzeichnen.

Judith Neumaier

Mok TS et al. Osimertinib or Platinum-Peme trexed in EGFR T790M-Positive Lung Cancer. N Engl J Med. 2017;376(7):629-40.

\section{NSCLC: Pembrolizumab in der Erstlinie}

\author{
In der Studie KEYNOTE-024 wurden der PD-1-Hemmer Pembrolizumab \\ und eine platinbasierte Chemotherapie in der Erstlinienbehandlung bei \\ Patienten mit fortgeschrittenem nichtkleinzelligem Lungenkarzinom \\ (NSCLC) und PD-L1-Expression in mindestens $50 \%$ der Tumorzellen \\ verglichen.
}

$\mathrm{n}$ die Phase-III-Studie KEYNOTE-024 wurden 305 Patienten aufgenommen, die ein bisher unbehandeltes fortgeschrittenes NSCLC mit PD-L1-Expression bei mindestens $50 \%$ der Tumorzellen und weder aktivierende Mutationen des EGFR-Gens noch Translokationen des ALK-Gens aufwiesen. Sie erhielten randomisiert Pembrolizumab (200 mg alle 3 Wochen) oder eine platinbasierte Therapie nach Wahl des Studienarztes (am häufigsten Carboplatin plus Premetrexed). Primärer Endpunkt war das progressionsfreie Überleben (PFS).

Nach median 11,2 Monaten war das mediane PFS unter Pembrolizumab signifikant länger als unter der Chemotherapie (10,3 vs. 6,0 Monate; Hazard
Ratio [HR] für Progression oder Tod 0,50, $95 \%$-Konfidenzintervall [95\%-KI] $0,37-0,68 ; \mathrm{p}<0,001)$. Nach 6 Monaten lebten noch $62,1 \%$ bzw. 50,3\% der Patienten ohne Progression. Der Nutzen von Pembrolizumab hinsichtlich des PFS war in allen Subgruppen nachweisbar. Auch die Gesamtüberlebensrate nach 6 Monaten war unter Pembrolizumab signifikant höher $(80,2$ vs. $72,4 \%$; HR für Tod 0,60; $p=0,005)$. Darüber hinaus war die Ansprechrate unter Pembrolizumab höher als unter der Chemotherapie $(44,8$ vs. $27,8 \%)$ und die mediane Dauer des Ansprechens länger (nicht erreicht vs. 6,3 Monate).

Therapiebedingte unerwünschte Ereignisse traten unter Pembrolizumab seltener auf als unter der Chemotherapie $(73,4$ vs. 90,0\%), ebenso therapiebedingte unerwünschte Ereignisse vom Grad 3-5 (26,6 vs. 53,3\%). Am häufigsten waren Diarrhö, Fatigue und Fieber unter Pembrolizumab sowie Anämie, Übelkeit und Fatigue unter der Chemotherapie. In der Pembrolizumab-Gruppe starb ein Patient an einem therapiebedingten unerwünschten Ereignis, in der Chemotherapiegruppe waren es 3 Patienten.

Fazit: Bei Patienten mit fortgeschrittenem NSCLC und PD-L1-Expression in mindestens $50 \%$ der Tumorzellen ging die Erstlinientherapie mit Pembrolizumab im Vergleich zur platinbasierten Chemotherapie mit einem signifikant längeren PFS einher. Auch die Rate an unerwünschten Ereignissen war unter dem PD-1-Hemmer niedriger.

Judith Neumaier

Reck $M$ et al. Pembrolizumab versus Chemotherapy for PD-L1-Positive Non-Small-Cell Lung Cancer. N Engl J Med. 2016;375(19):1823-33. 